\title{
Endothelial Regulator of Calcineurin 1 Promotes Barrier Integrity and Modulates Histamine-Induced Barrier Dysfunction in Anaphylaxis
}

OPEN ACCESS

Edited by:

Miriam Wittmann,

University of Leeds, United Kingdom

Reviewed by:

Vineesh Vimala Raveendran, King Faisal Specialist Hospital \& Research Centre, Saudi Arabia

Katherine R. Martin,

Institut Cochin (INSERM), France

*Correspondence: Vanesa Esteban vesteban@fjd.es

Specialty section:

This article was submitted to Inflammation,

a section of the journal

Frontiers in Immunology

Received: 24 April 2017 Accepted: 29 September 2017 Published: 20 October 2017

Citation:

Ballesteros-Martinez C, Mendez-

Barbero N, Montalvo-Yuste A, Jensen BM, Gomez-Cardenosa A,

Klitfod L, Garrido-Arandia M, Alvarez-Llamas G, Pastor-Vargas C, Vivanco F, Garvey LH, CuestaHerranz J, Poulsen LK and Esteban V (2017) Endothelial Regulator of Calcineurin 1 Promotes Barrier Integrity and Modulates HistamineInduced Barrier Dysfunction in Anaphylaxis.

Front. Immunol. 8:1323. doi: 10.3389/fimmu.2017.01323

\begin{abstract}
Constanza Ballesteros-Martinez', Nerea Mendez-Barbero², Alma Montalvo-Yuste', Bettina M. Jensen ${ }^{3}$, Aída Gomez-Cardenosa ${ }^{4}$, Lotte Klitfod ${ }^{5}$, María Garrido-Arandia ${ }^{6}$, Gloria Alvarez-Llamas', Carlos Pastor-Vargas ${ }^{1}$, Fernando Vivanco', Lene Heise Garvey ${ }^{3}$, Javier Cuesta-Herranz ${ }^{1,4}$, Lars K. Poulsen ${ }^{3}$ and Vanesa Esteban ${ }^{1,3 *}$

'Department of Immunology, IIS-Fundación Jiménez Díaz, Autonomous University of Madrid, Madrid, Spain, ${ }^{2}$ Department of Vascular Physiopathology, IIS-Fundación Jiménez Díaz, Autonomous University of Madrid, Madrid, Spain, ${ }^{3}$ Allergy Clinic, Gentofte Hospital, Copenhagen University Hospital, Hellerup, Denmark, ${ }^{4}$ Department of Allergy, Fundación Jiménez Díaz, Madrid, Spain, ${ }^{5}$ Surgery Department, Gentofte Hospital, Copenhagen University Hospital, Hellerup, Denmark, ${ }^{6}$ Center for Plant Biotechnology and Genomics, Technical University of Madrid, Madrid, Spain
\end{abstract}

Anaphylaxis, the most serious and life-threatening allergic reaction, produces the release of inflammatory mediators by mast cells and basophils. Regulator of calcineurin 1 (Rcan1) is a negative regulator of mast-cell degranulation. The action of mediators leads to vasodilation and an increase in vascular permeability, causing great loss of intravascular volume in a short time. Nevertheless, the molecular basis remains unexplored on the vascular level. We investigated Rcan1 expression induced by histamine, platelet-activating factor (PAF), and epinephrine in primary human vein (HV)-/artery (HA)-derived endothelial cells (ECs) and human dermal microvascular ECs (HMVEC-D). Vascular permeability was analyzed in vitro in human ECs with forced Rcan1 expression using Transwell migration assays and in vivo using Rcan1 knockout mice. Histamine, but neither PAF nor epinephrine, induced Rcan1-4 mRNA and protein expression in primary HV-ECs, HA-ECs, and HMVEC-D through histamine receptor 1 (H1R). These effects were prevented by pharmacological inhibition of calcineurin with cyclosporine A. Moreover, intravenous histamine administration increased Rcan1 expression in lung tissues of mice undergoing experimental anaphylaxis. Functional in vitro assays showed that overexpression of Rcan 1 promotes barrier integrity, suggesting a role played by this molecule in vascular permeability. Consistent with these findings, in vivo models of subcutaneous and intravenous histamine-mediated fluid extravasation showed increased response in skin, aorta, and lungs of Rcan1-deficient mice compared with wild-type animals. These findings reveal that endothelial Rcan1 is synthesized in response to histamine through a calcineurin-sensitive pathway and may reduce barrier breakdown, thus contributing to the strengthening of the endothelium and resistance to anaphylaxis. These new insights underscore its potential role as a regulator of sensitivity to anaphylaxis in humans.

Keywords: anaphylaxis, endothelial cells, vascular permeability, regulator of calcineurin 1, histamine 


\section{INTRODUCTION}

Anaphylaxis is a potentially lethal, rapid-onset allergic reaction, and is known to be the most aggressive manifestation of allergic disorders (1). Anaphylactic reactions trigger a broad range of symptoms affecting different organs and bodily systems. The severe alterations described in human anaphylaxis involve the cardiovascular system and include loss of peripheral vascular resistance (vasodilation) and exacerbated vascular extravasation. These physiological processes are associated with low blood pressure, reduced venous return, and decreased cardiac output. Furthermore, bronchoconstriction and pulmonary/coronaryartery vasoconstriction commonly occur in the thoracic cavity, which contributes to widespread circulatory collapse (2-4). The clinical features of anaphylaxis have been well-described and classified, though investigations into its molecular signaling dynamics remain scarce in humans. To date, this hypersensitivity event has been considered a disorder of the immune system. However, immunological mechanisms do not fully explain the versatility of the events that take place during a reaction (5). In order to obtain a better understanding of anaphylaxis, we searched for new strategies, including the study of vascular mechanisms.

Molecules released mainly by active mast cells and basophiles interact with the vascular endothelium and the smooth muscle layers, destabilizing the endothelial barrier and modifying essential vascular contractile functions (tone) in vessels and airways (6). Tryptase, histamine, and platelet-activating factor (PAF) are only two such relevant biochemical mediators found in the serum of anaphylaxis patients (7). In cells, histamine binds to four types of receptors coupled to $\mathrm{G}$ proteins $(\mathrm{H} 1-4 \mathrm{R})$, and these receptors are widely distributed across tissues and leads to signaling through numerous molecular pathways. Type- 1 and type-2 histamine receptors (H1R and $\mathrm{H} 2 \mathrm{R}$ ) have been widely investigated due to the fact that they mediate the intracellular signaling associated with second messengers such as $\mathrm{Ca}^{2+}$ and cAMP, which regulate vascular permeability, vasodilation, and bronchoconstriction $(8,9)$. In order to control these homeostatic disorders, the first-line treatment for anaphylaxis is intramuscular injection of epinephrine, which acts via $\alpha$ - and $\beta$-adrenergic receptors, triggering intracellular mechanisms in cardiac and smooth vascular cells (10). Their potent vasoconstrictor actions combined with fluid therapy are effective when administered promptly (11). Moreover, the second messenger CAMP is mediated by activation of $\beta$-adrenergic receptor signaling and contributes to the maintenance of endothelial barrier properties under baseline conditions (12).

There is growing interest in understanding the vascular permeability and vasodilation that occur during anaphylaxis. Different endothelial molecular pathways have been described as key targets for anaphylaxis due to their implication in the disruption of endothelial integrity or vascular tone modulation $(13,14)$. In humans, changes in vascular permeability during anaphylaxis may lead to a transfer of $50 \%$ of the intravascular fluid into the interstitial space within $10 \mathrm{~min}$ (3). At the molecular and cellular level, it is well known that histamine induces rapid and transient processes, which disrupt the endothelial barrier, thereby allowing the leakage of fluids, mainly in venules (15). Furthermore, endothelial cells (ECs) participate in physiological processes that regulate not only the capillary component but also peripheral vascular resistance and homeostasis. This fact is a focus of research in vascular permeability modulation (16), and vascular wall components are also essential in regulating leakage and peripheral vascular resistance in anaphylaxis. Mechanistically, a cellular counterbalance between contractile and adhesive forces must exist to maintain the stability between cells and prevent the rupture of the endothelial barrier (17). It has been reported that ECs contribute to the widespread effects observed in anaphylaxis through synthesis and the release of substances, including nitric oxide (NO) and mediators generated from the arachidonic acid cascades (18). However, mast cells are the main cellular source recognized to date, and are major releasers of prostaglandins and leukotrienes eliciting anaphylaxis reactions $(19,20)$.

Histamine binding to $\mathrm{H} 1$ receptors activates PLC $\beta$ and elevates intracellular $\mathrm{Ca}^{2+}$, both of which determine the signaling pathways which regulate inflammatory processes. $\mathrm{Via} \mathrm{Ca}^{2+}$-dependent mechanisms, changes take place in cytoskeleton proteins or junction structures that determine cellular permeability and contractility (21). One of the most sensitive downstream effectors of $\mathrm{Ca}^{2+}$ is the ubiquitously expressed serine/threonine protein phosphatase calcineurin (22). Activation of calcineurin contributes to immune response signaling by members of the nuclear factor of activated T-cells (NFAT) family (23). Calcineurin activity can be inhibited by the immunosuppressant cyclosporine A (CsA) which forms a complex with cyclophilin A to bind and competitively inhibit calcineurin phosphatase activity (24). Endogenous regulation of calcineurin is mediated by members of the regulator of calcineurin (Rcan) family, and Rcan 1 is the only such molecule regulated by $\mathrm{Ca}^{2+}$ /calcineurin (25). The RCAN1 gene contains seven exons that can generate several transcripts resulting from differential promoter use and first exon choice. The two major transcriptional products for Rcan 1 are isoforms, including exons $1+5-7$ (Rcan11 ) and isoform 4 (Rcan1-4) with exons $4+5-7$, which produce proteins with 252 and 197 amino acids, respectively $(26,27)$.

Divergent functions have been reported for both Rcan1-1 and Rcan1-4. While different inducers of $\mathrm{Ca}^{2+}$ selectively upregulate Rcan1-4, few stimuli have been described as modulators of Rcan1-1 expression. A role for apoptosis is attributed to Rcan1-1 in response to glucocorticoids, and relevant studies have linked Rcan1-1 to Huntington disease $(28,29)$. Rcan1-4 is upregulated by increases in $\mathrm{Ca}^{2}$ or in response to a variety of signals, including cytokines, hormones, hydrogen peroxide, and stress (30). Functionally, it has been widely described as an anti-inflammatory, anti-angiogenic agent and modulator of cardiovascular pathologies $(31,32)$. Due to its influence in regulating calcineurin activity, Rcan 1 is involved in a broad range of cellular systems and biological processes. Extensive investigations have provided insights into EC signaling, describing Rcan 1 as a potential therapeutic target in vascular inflammation (33). VEGF and thrombin have been reported to be the major inducers of Rcan 1 in ECs, while angiotensin II induces Rcan 1 expression in vascular smooth muscle cells (34-36).

Given the crucial role exerted by mediators on the vascular wall in anaphylaxis, we assessed the impact of anaphylaxis on Rcan1 expression in human ECs, as well as its functional involvement in vascular permeability and cell dilation. This study evaluates 
Rcan1 expression in human ECs in response to mediators of anaphylaxis and, more specifically, the involvement of histamine receptors involved in Rcan1 expression. Moreover, we studied the permeability effects of Rcan1 causing either endothelial barrier rupture or strengthening in response to mediators of anaphylaxis and also analyzed the plausible endothelial mechanisms exerting these functions.

\section{MATERIALS AND METHODS}

\section{In Vitro Human Cell Cultures}

Human dermal microvascular ECs (HMVEC-D) were acquired from Lonza. Human vascular endothelial vein and artery cells were isolated from the macroscopically healthy part of intact saphenous veins harvested from patients undergoing bypass surgery/high ligation of varicose veins. The study was approved by the research ethics committees of the Gentofte and FJD hospitals, and written informed consent was obtained from all patients. Briefly, after removal of the connective tissue, the vein was opened longitudinally and the endothelium was isolated by digestion with $0.1 \%$ type I collagenase (Gibco) in PBS for $30 \mathrm{~min}$ at $37^{\circ} \mathrm{C}$. Similarly, artery specimens were incubated with the digestion buffer O.N at $37^{\circ} \mathrm{C}$. Reactions were stopped and cells were collected by centrifugation and grown in DMEMF12 media supplemented with $10 \mathrm{U} / \mathrm{ml}$ heparin, $30 \mathrm{~g} / \mathrm{ml} \mathrm{ECGF}$, $100 \mathrm{U} / \mathrm{ml}$ penicillin, $100 \mathrm{~g} / \mathrm{ml}$ streptomycin, and $15 \%(\mathrm{v} / \mathrm{v})$ heat-inactivated fetal bovine serum. Cells were seeded in a plate previously coated with $0.5 \%$ sterile gelatin and maintained in a humidified atmosphere of $5 \% \mathrm{CO}_{2}$ in air at $37^{\circ} \mathrm{C}$. After $5-7$ days of incubation, human vein or artery ECs (abbreviated herein as HV-EC/HA-ECs) were selected with human CD31 antibody. Next, the secondary antibody associated with magnetic beads (Dynabeads ${ }^{\circledR}$ anti-mouse IgG from CELLection TM Pan Mouse IgG Kit) was incubated for $30 \mathrm{~min}$ at $4^{\circ} \mathrm{C}$ with constant shaking. ECs were seeded on plates previously treated with $0.5 \%$ gelatin. Once in confluence, primary cell cultures were FBS 0.5\% starved for $18 \mathrm{~h}$ before the experiments were performed. All experiments were performed during passages 3-7. A similar protocol was applied to the artery specimens.

\section{Reagents}

Histamine, PAF, epinephrine, and the histamine receptor antagonists diphenhydramine hydrochloride (H1RB) and famotidine (H2RB) were obtained from Sigma.

\section{Protein Extraction and Immunoblot Analysis}

Endothelial cells were lysed with buffer containing Tris, $\mathrm{NaCl}$, EDTA, EGTA, Triton X-100, NP40, protease inhibitors, PMSF, and DTT. Cellular lysates were shaken for $15 \mathrm{~min}$ and centrifuged at $12,000 \mathrm{rpm}$ at $4^{\circ} \mathrm{C}$. After stimulation with reagents or sera, ECs were washed with ice-cold PBS and protein extracts obtained as previously described (36). Rabbit polyclonal anti-Rcan 1 primary antibody was used at a ratio of 1/1,000 (Sigma). Analysis of images was carried out using the ImageJ program. Protein extracts from animal tissues were previously disaggregated and processed in similar fashion.

\section{mRNA Extraction, RT, and Real-time PCR Analysis}

RNA extraction was performed using Tri Reagent (Molecular Research Center). Two micrograms of total RNA were reverse transcribed following the instructions for the high-capacity cDNA RT (Thermo Fisher) protocol, and samples were stored at $-70^{\circ} \mathrm{C}(36)$. The following TaqMan FAM/MGB probes were purchased from Applied Biosystems: human Rcan1 (Hs01120954_m1), human Rcan1.1 (Hs01120956_m1), human PTGS2 (Hs00153133_m1), human MYLK (Hs00364926_m1), human NOS3 (Hs01574659_m1), human ROCK1 (Hs01127699_m1), and human CAMK2B (Hs00365799_m1). Human Rcan1.4 was purchased customized according to primer forward: GCAAACAGTGATATCTTCAG CGAAA, primer reverse: GTGATGTCCTTGTCATACGTCCTAA, and the labeled CAGGGCCAAATTT (5 NFQ). Reactions were incubated in the presence of AmpliTaq Gold DNA polymerase (Applied Biosystems) for $2 \mathrm{~min}$ at $50^{\circ} \mathrm{C}$ followed by $10 \mathrm{~min}$ at $95^{\circ} \mathrm{C}$. Reactions were then run over 40 cycles of $95^{\circ} \mathrm{C}$ for $15 \mathrm{~s}$ and $60^{\circ} \mathrm{C}$ for $1 \mathrm{~min}$. Human beta actin VIC/MGB or $18 \mathrm{~s}$ VIC/MGB rRNA transcripts were used as an internal control, which were amplified in the same tube to normalize for variation in input RNA. The amount of target mRNA in the samples was estimated by the 2CT relative quantification method. Ratios were calculated between the amounts of mRNA from stimulated and/or transfected vs nonstimulated control ECs.

\section{In Vitro Vascular Permeability Assays}

Endothelial barrier integrity was evaluated by using Transwell 24-well cell culture inserts (TW) including a membrane pore size of $0.4 \mu \mathrm{m}$ (Corning). ECs were seeded at a density of $10^{5}$ cells/ well in TWs previously coated with $0.5 \%$ gelatin diluted in sterile water and grown in DMEMF12 media supplemented as described above. After several days, EC monolayers were observed and starved for $18 \mathrm{~h}$ before the experiments were performed. Stimulus together with a final concentration of $1 \mathrm{mg} / \mathrm{ml}$ of FITC-Dextran (Sigma) was added to the upper chamber. Vascular endothelialpermeability measurements were determined by measuring fluorescence of the recipient at 5-30 $\mathrm{min}$ and $2 \mathrm{~h}$. All samples were evaluated at least in duplicate.

\section{Lentiviral Production and Infection}

Lentiviruses expressing Rcan1-1-IRES-GFP, Rcan1-4-IRES-GFP, and IRES-GFP were obtained by transient calcium phosphate transfection of HEK-293. The supernatant containing the lentiviral particles was collected $48 \mathrm{~h}$ after removal of the calcium phosphate precipitate, filtered through a $45-\mu \mathrm{M}$ PVDF membrane (Steriflip; Millipore), and ultracentrifuged for $2 \mathrm{~h}$ at $26,000 \mathrm{rpm}$ at $4^{\circ} \mathrm{C}$ (Ultraclear Tubes, SW28 rotor, and Optima 1-100 XP Ultracentrifuge; Beckman Coulter). Viruses were resuspended, titrated, and infection efficiency (GFP-expressing cells) was monitored by flow cytometry (36). Lentiviral infection was performed in subconfluent primary cultures of ECs with a 1:1 mix including Rcan1-IRES-GFP (Rcan1-1-IRES-GFP + Rcan14-IRES-GFP) or IRES-GFP. Cells were exposed to the lentiviruses in the presence of $10 \%$ FBS during $5 \mathrm{~h}$ (37). After 4 days, cells were starved of FBS $(0.5 \%)$ during for $18 \mathrm{~h}$ and the experiments of interest were performed. 


\section{Animal Experimental Designs}

Animal procedures were carried out in accordance with the European Union guidelines for the care and experimental use of animals. Protocols with reference PROEX: 391/15 received prior approval from the IIS-FJD Ethics Committee and the competent authorities in the region of Madrid.

Two-month-old C57BL/6J mice were intravenously (i.v.) injected with Evans blue $(0.04 \mu \mathrm{g} / \mathrm{g}$ in $\mathrm{NaCl})$ followed by subcutaneous (s.c.) injection with histamine and PAF at 5-50 ng/ml for $10 \mathrm{~min}$. To evaluate systemic vascular permeability, histamine at $10 \mathrm{mg} / \mathrm{kg}$ or PAF $2 \mu \mathrm{g} / \mathrm{g}$ were i.v. injected together with Evans blue dye. Once i.v. administrated, mice were sacrificed after $15 \mathrm{~min}$, and most PAF animals died spontaneously. The skin pieces, aortas, lungs, and hearts of these animals were incubated in $500 \mu \mathrm{l}$ of formamide at $55^{\circ} \mathrm{C}$ for $48 \mathrm{~h}$, and the Evans blue content was determined by absorption at $595 \mathrm{~nm}$. To test passive systemic anaphylaxis (PSA) using an experimental in vivo model, mice were i.v. injected with $20 \mu \mathrm{g}$ of anti-DNP IgE. After $24 \mathrm{~h}$, the mice were challenged with an i.v. injection of $1 \mathrm{mg}$ DNP-HSA (human serum albumin) for the development of anaphylaxis. To test for active systemic anaphylaxis (ASA), we used the classical model, sensitizing mice with i.p injection of $1 \mathrm{mg}$ BSA and $300 \mathrm{ng}$ pertussis toxins as adjuvant in normal saline. After 14 days, the mice were challenged with i.v. injection of $2 \mathrm{mg}$ BSA (38). Following administration of s.c. and i.v. histamine, PSA and ASA mice were sacrificed $30 \mathrm{~min}$ after the challenge by cervical dislocation, and blood sampling and biopsies (organ collection) were collected for molecular analysis. All studies were repeated at least once to assure reproducibility. Rcan1-deficient (-/-) mice were generated as previously described (39). All mice were genotyped by PCR of tail samples using the following primers: Rcan1, 5'-GGTGGTCCACGTGTGTGAGA-3', 5'-ACGTGAACAAAG GCTGGTCCT-3', and 5'-ATTCGCAGCGCATCGCCTTCTAT CGCC- $3^{\prime}$. Control littermates were used in all experiments.

\section{Statistical Analysis}

All values are expressed as the mean \pm SEM. Differences were evaluated with GraphPad Prism 7.3 program using one-way ANOVA analysis followed by Bonferroni's post hoc test (experiments $\geq 3$ groups) or Student's $t$-test (experiments with two groups). Statistical significance was set at $p<0.05$.

\section{RESULTS}

\section{Histamine Increases Rcan1-4 Expression in HV-EC}

Previous studies have demonstrated the stimulation of Rcan1-4 protein induced by agents that cause the mobilization of $\mathrm{Ca}^{2+}$ $(33,34,36)$. We observed that histamine stimulation induces a specific increase in Rcan1-4 but not Rcan1-1 protein after a short period (30 min of stimulation); the increase was considerably greater after $60 \mathrm{~min}$ of exposure in HV-ECs (Figures 1A,B). Parallel to these results, Rcan 1-4 mRNA expression also increased during the same time frame in response to histamine (Figure 1C). Moreover, as histamine is not the sole mediator recognized for anaphylaxis, we addressed the effect of PAF on HV-ECs. Over the last decade, evidence based primarily on animal models has indicated the existence of an anaphylaxis reaction mediated by IgG- FcyRIII $(5,40)$, and PAF has been recognized as essential in those non-IgEmediated reactions (41). In our studies, PAF did not modify Rcan1-4 protein expression in HV-ECs (Figures 1A,B). Additionally, since epinephrine/adrenaline is the first-line treatment in anaphylaxis $(1,42)$, we set out to determine whether epinephrine could modulate Rcan1-4 expression in ECs. Incubation of epinephrine from $15 \mathrm{~min}$ to $1 \mathrm{~h}$ did not modify Rcan 1-4 protein expression; however, histamine and epinephrine coincubation increased the expression of the Rcan1-4 protein in HV-ECs (Figures 1D,E). As with the protein findings, Rcan 1 mRNA expression increased in response to histamine and epinephrine (Figure 1F). In all cases, the Rcan1-1 protein and mRNA isoform was unchanged in its expression.

\section{Histamine Increases Rcan1-4 Expression in HA-EC, HMVEC-D, and Lungs of Experimental Anaphylaxis}

The cellular heterogeneity of the whole endothelial compartment has been recognized for some time. Both intracellular mechanisms and functional abilities may be different between the micro-, artery, or vein ECs $(43,44)$. For this reason, we next checked Rcan1-4 modulation by histamine and epinephrine in other endothelial vascular microenvironments. By using primary artery-derived ECs (HA-EC), our results showed that the contact with histamine induced a marked increase in Rcan 1-4 protein expression after $30 \mathrm{~min}$ and $1 \mathrm{~h}$ (Figures 2A,B), which was correlated with elevated levels of Rcan1-4 mRNA expression (data not shown). As we previously observed in HV-ECs, epinephrine had no effect on Rcan1-4 expression when incubated alone; however, coincubation with histamine induced a high Rcan1-4 increase in HA-ECs. Additionally, experiments addressing Rcan 1 expression in human dermal microvascular ECs (HMVEC-D) showed similarly increased levels of Rcan1-4 protein and mRNA expression in response to histamine. Contrary to this, no effect was observed upon epinephrine stimulation within the studied time frame in HMVEC-D (Figures 2C,D). These results suggest that histamine modulates Rcan1-4 expression in ECs of large vessels regardless of whether they are veins, arteries, or the microvasculature.

The molecular and physiological differences found between studies in animals and studies in humans have sparked debate in anaphylaxis as well as other fields of research (5). Using three experimental designs simulating different degrees of allergic sensitivity, we looked for Rcan1 expression in target organs in mice undergoing anaphylaxis. Experimental passive anaphylaxis (PSA) is based on systemic IgE anti-DNP sensitization over $24 \mathrm{~h}$, while ASA was induced by BSA followed by challenge 14 days later. Analysis of Rcan 1 expression in lung extracts of mice treated with histamine for $30 \mathrm{~min}$ or undergoing PSA or ASA showed increased Rcan1-4 levels compared to lungs of control mice (Figures 2E,F).

\section{Rcan1-4 Protein Expression Is Induced by Histamine via Its H1 Receptor and a Calcineurin-Dependent Mechanism}

Studies were performed to evaluate the relevant receptors (H1R/ $\mathrm{H} 2 \mathrm{R}$ ) involved in histamine-induced Rcan1-4 expression. 




Prior to stimulation with histamine, HV-ECs were incubated with increasing concentrations of diphenhydramine hydrochloride (type 1 receptor antagonist), determining the optimal concentration of use at $10^{-5} \mathrm{M}$. Cellular pre-incubation with diphenhydramine hydrochloride completely blocked Rcan1-4 expression induced by histamine in HV-ECs (Figures 3A-C). In contrast, no inhibitory effect mediated by famotidine (type 2 receptor antagonist) was observed; indeed, an additional increase in Rcan1-4 expression induced by histamine stimulation was noted when the receptor type 2 was blocked (Figures 3A-D).
Additionally, coincubation with antagonists for both receptors (diphenhydramine hydrochloride plus famotidine) abolished Rcan 1-4 expression induced by histamine in HV-EC and HA-ECs (Figures 3C,D).

Next, we investigated the potential role of calcineurin in mediating Rcan1-4 expression induced by histamine. Cellular pre-incubation with CsA completely blocked Rcan1-4 expression induced by histamine in HV-ECs and HMVEC-D (Figures 3E,F). Similar to the protein findings, Rcan 1-4 mRNA expression was significantly diminished in the presence of CsA (Figure 3G). 


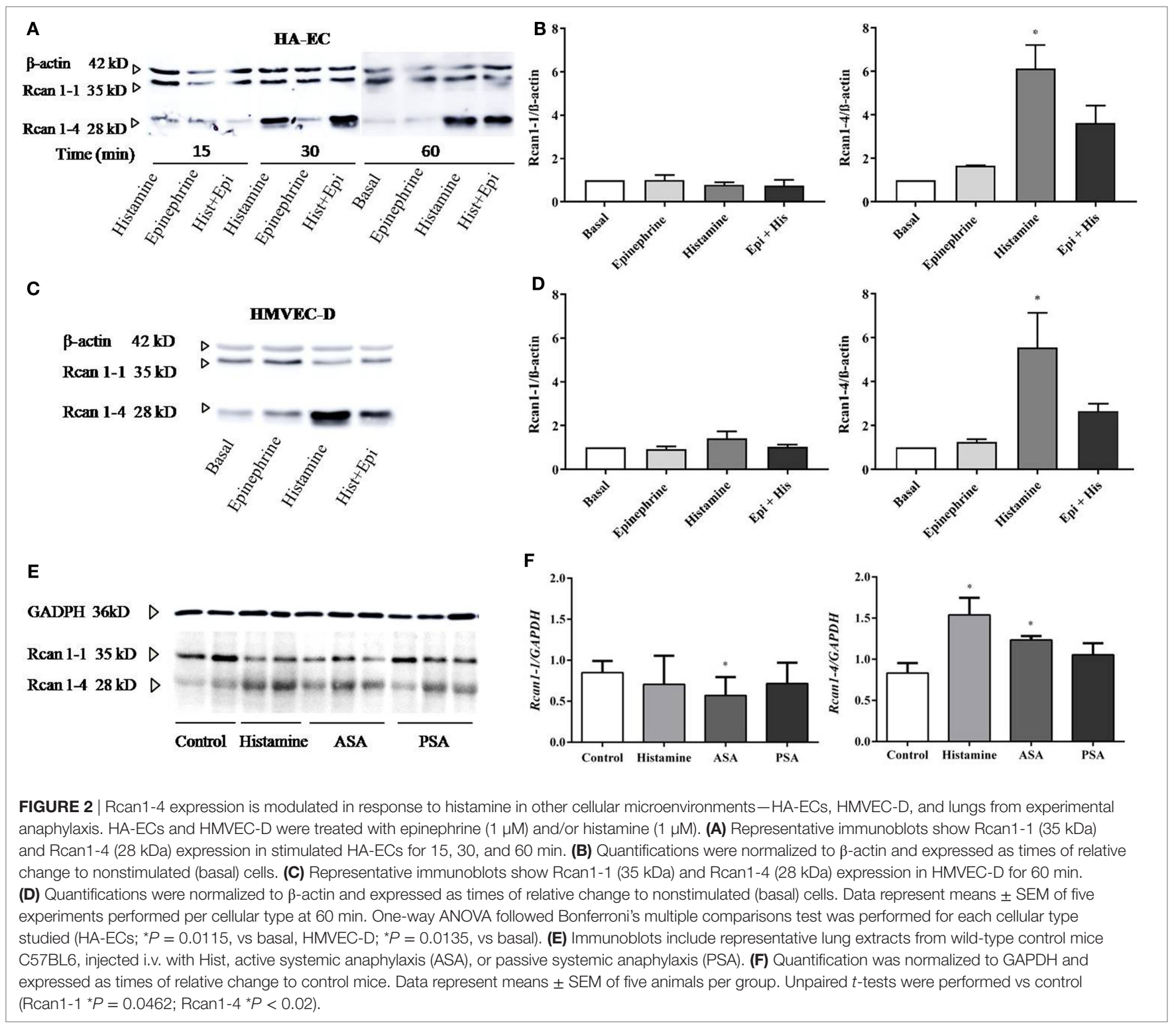

In addition, an increase in calcineurin phosphatase activity is observed in response to histamine in HMVEC-D (data not shown).

\section{Human Rcan1 Induces Marked Prevention in Vascular Permeability, Strengthening the Endothelial Contact}

In vitro ECs adhere to coated surfaces, making up the endothelial monolayer. To evaluate vascular permeability in vitro, we employed available assay systems (Transwell, TW) based on measurements of extravasations of fluids through an endothelial monolayer. These cells supported on permeable membranes are inserted in individual containers that allow the transport of molecules through them (Figure 4A). First, it was verified that the system allows a measurable increase of FITC-Dextran over time. EC monolayer blocks the passage of the dye in resting conditions (and only endothelial permeability is increased throughout the course of the experiment). As expected, short incubations (minutes) with the vasoactive mediators histamine and PAF induced a rapid increase in vascular permeability on HMVEC-D, which was not observed by incubation with epinephrine (12). The response duration and sensitivity induced by histamine may vary depending on ECs (45). After 15-30 min, we observed a clear tendency to block the passage of molecules across the endothelial barrier both in the presence of histamine and epinephrine, indicating a transient effect of histamine on vascular permeability followed by cell dilation. At this time, only PAF continued destabilizing the endothelial barrier (Figure 4B). Following this, we checked the histamine-induced barrier effects in ECs of big vessels. Our studies did not show modification in barrier properties after short periods of stimulation with histamine in HV-ECs or HA-ECs. However, a clear cell-dilating effect induced by histamine is 


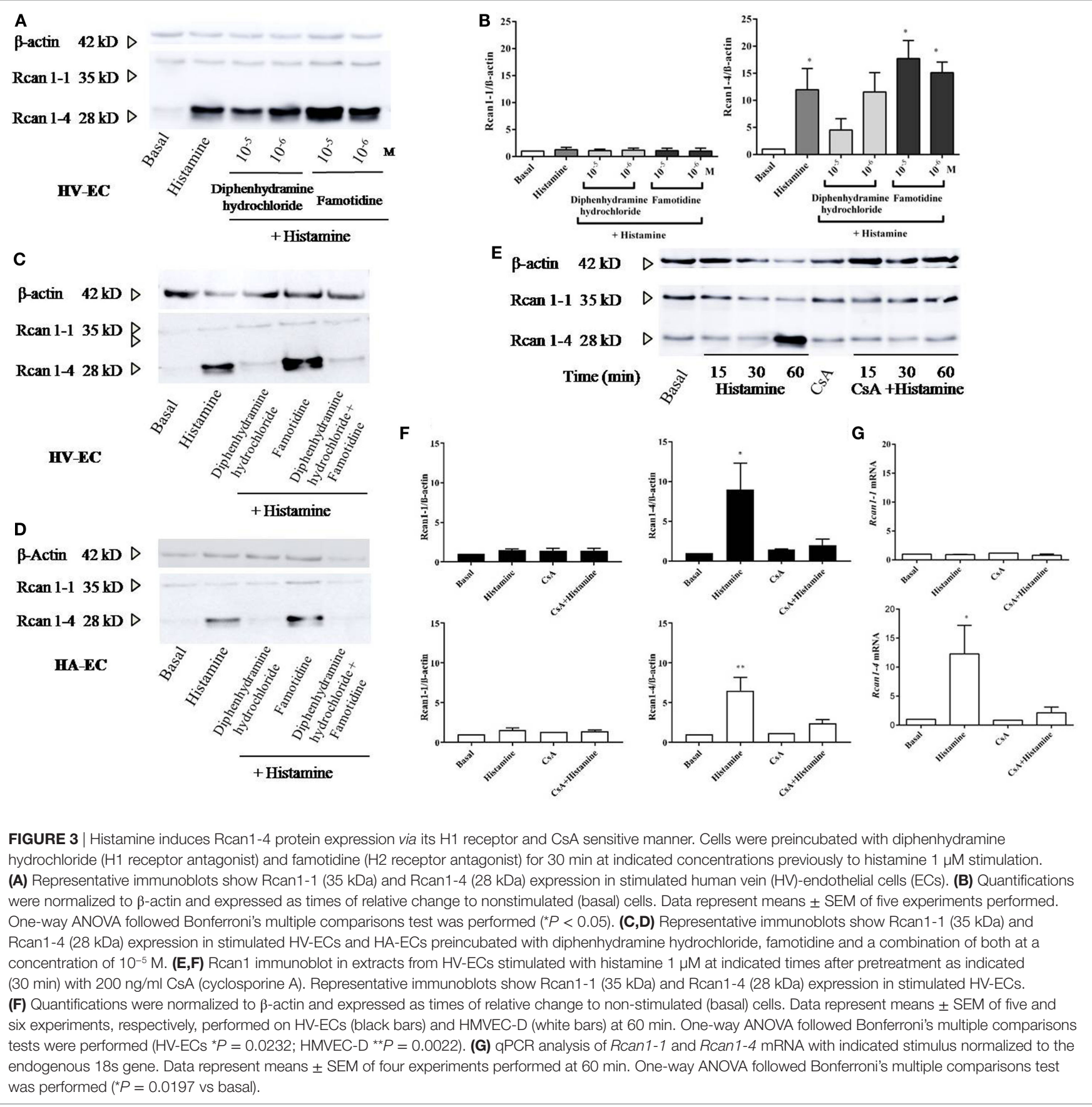

observed in HV-ECs, and a tendency toward this same dynamic was seen in HA-ECs (Figure 4C). In order to determine whether Rcan 1 has a functional contribution in these processes, we used lentiviral constructs aimed at exogenously modifying Rcan1 expression on ECs. HV-ECs were transduced with Rcan1IRES-GFP or the lentiviral control IRES-GFP. The efficiency of infection in human cell cultures was analyzed by GFP expression using flow cytometry (data not shown), fluorescence microscopy, and Western blotting (Figure 4D). Lentiviral infection does not modify the effects of histamine on cells, as the Rcan1 pattern expression showed the increase of Rcan 1-4 protein resembling that seen in non-transduced cells for both IRES-GFP and Rcan1IRES-GFP HV-ECs (Figures 1-3).

To evaluate the role of Rcan 1 in vascular permeability, IRESGFP and Rcan1-IRES-GFP HV-ECs were seeded in TW and stimulated with or without histamine for $30 \mathrm{~min}$. Data show that Rcan1 overexpression prevents basal FITC extravasation in HV-ECs, indicating a marked role of Rcan 1 in blocking cell permeability. In addition, HV-ECs infected with control vector and exposed to histamine produced an effect resembling the one observed previously in non-transduced cells. However, no additional effects of histamine were observed when the cells 


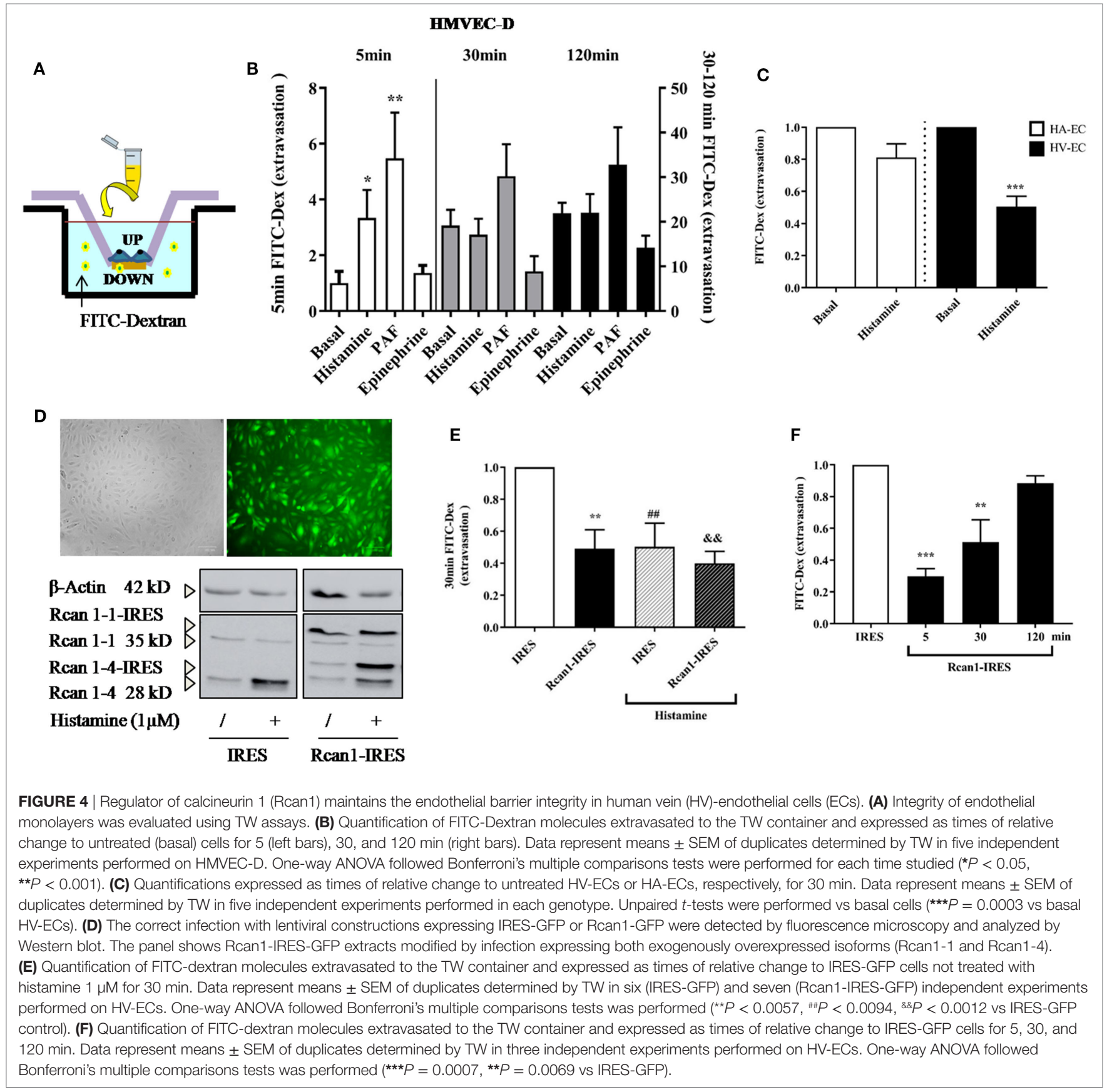

overexpressed Rcan 1 endogenously (Figure 4E). To determine whether Rcan 1 can also affect the earliest phases of extravasation and its stability over time, time frames of 5 and $120 \mathrm{~min}$ were used for the measurements (Figure 4F). Our results indicate that Rcan1 stabilizes the endothelial barrier during short incubation times, though this effect is weakened when incubation is longer.

We, therefore, speculated that increased expression of Rcan 1 in ECs could in turn modulate other primordial factors involved in anaphylaxis. To test this hypothesis, we analyzed the expression of some genes involved in vascular homeostasis and barrier stability: cyclooxygenase-2 (COX-2), calmodulin-dependent protein kinase II-dependent (CaMKII), nitric oxide synthase 3 (NOS3), MLC-kinase (MLCK), and Rho-kinase 1 (ROCK1) in resting and histamine-stimulated IRES-GFP and Rcan1-IRESGFP HV-ECs. Resting Rcan1-IRES-GFP HV-ECs showed a marked inhibition of COX-2 mRNA, CaMKII mRNA, and NOS3 expression. However, similar levels of $M L C K$ and ROCK1 were observed compared to IRES-GFP HV-ECs (Figure 5). This brief screening suggests that consequent generation of newly formed proinflammatory phospholipid-endothelial derived COX-2 could contribute to vascular contractile processes concomitant to 
anaphylactic reactions. Additionally, a direct effect on the production of the NO is exerted when Rcan 1 is overexpressed.

\section{Mouse Rcan1-/- Tissues Present Higher Leakage in Response to s.c. and i.v. Histamine Administration}

The in vitro results described above led us to investigate the role of Rcan 1 in experimental vascular permeability in vivo. We examined the effect exerted by histamine and PAF on vascular permeability in Rcan1-deficient vs $W T$ mice. $\mathrm{NaCl}$ s.c. injection does not modify basal extravasation in WT and Rcan $^{-/-}$mice (data not shown). However, an increase of leakage was seen in response to s.c. injection of histamine in both types of mice, and this increase was dose-dependent and significantly higher in $\mathrm{Rcan}^{-/-}$mice (Figures 6A,B). Moreover, histamine administrated intravenously increased extravasation in aortas and lungs, though not in the hearts of $R c a n 1^{-/-}$mice, while no significant difference was observed in response to PAF between genotypes (Figures 6B,C). As in the in vitro human cells, endothelial Rcan 1 may prevent or recover the loss of endothelial barrier function induced by histamine.

\section{DISCUSSION}

The functional heterogeneity between different EC niches and their importance in the release of certain physiological factors contributing to pathological situations is a topic of high relevance in important fields of research $(16,46)$. Therefore, in anaphylaxis affecting a large number of organs, it is plausible that the vascular system is a critical participant in the evolution of symptoms and one that may condition the progress of reactions. This study provides the first evidence that histamine, a relevant mediator involved in anaphylactic reactions, modulates Rcan1-4 expression in different human vascular niches (HV-ECs, HA-ECs, and HMVEC-D). Different authors have previously reported that factors such as VEGF and thrombin induce Rcan1-4 expression in cultured ECs $(34,47)$. Given the major role of histamine when released by immune cells as a mediator of anaphylactic reactions, our results extend previous findings, showing that histamine upregulates Rcan1-4 expression in several types of ECs, whereas Rcan1-1 is not modulated. In agreement with our findings, a number of studies performed on this cellular type did not observe significant Rcan1-1 variation in response to stimuli (34) in spite of its involvement in Huntington disease and mitochondrial autophagy $(29,48)$. Additionally, epinephrine has no effect on Rcan1 expression, and although its potent actions as a dilator or constrictor through its adrenergic receptors have been widely observed, its role in the endothelium has been poorly investigated. However, the contributions of epinephrine to the maintenance of the endothelial barrier, through its $\beta$-adrenergic receptors, have been evaluated in ECs (12). Similarly, a substantial number of studies have demonstrated the role of PAF in anaphylaxis. The data shown here do not support a role for PAF in modulating Rcan 1-4 expression in ECs, at least in our experimental conditions. However, given the relevance of PAF, more thorough studies focused on human vascular cells would be of interest to clarify unexplored aspects that thus far have been mostly related to reactions mediated by IgE or IgG antibodies in anaphylaxis $(5,41)$.

The human H1R acts mainly by coupling to $\mathrm{Gq} / 11$ proteins; in fact, experimental anaphylaxis is prevented in endothelial Gq/11deficient mice (38). Through its specific signal, histamine is one of the most potent vasoactive substances, inducing relaxation or contraction (tissue- and species-dependent) and participating in anaphylactic responses, mainly through its $\mathrm{H} 1 \mathrm{R}$ and $\mathrm{H} 2 \mathrm{R}$ receptors $(8,9)$. The data shown here demonstrate that histamine increases Rcan 1 expression through the H1R receptor in ECs due to a calcineurin-dependent mechanism, as the calcineurin inhibitor CsA inhibits its expression.

Anaphylaxis is widely recognized by the presence of increased vascular permeability, mediating a shift of intravascular fluid into the extravascular space within minutes, which results in hypotension and hemoconcentration (3). Some studies in human subjects have revealed transient impairment of the microvasculature during severe acute anaphylaxis (49). Thus, the leakage occurring in anaphylaxis requires cellular retraction of ECs as a result of increased cell contractile pathways in response to external stimuli or agents (15). Subsequently, a biological counterbalance between contractile and adhesive forces must exist to maintain the stability between cells or allow cells to recover from the rupture of the endothelial sheet. Thus, most of the mediators described in anaphylaxis elicit vascular EC permeability signaling through specific receptors and molecular pathways. However, some stabilizing molecules preserve the rupture of the endothelial barrier (as cAMP or sphingosine 1 phosphate). The functional evaluation of Rcan 1 by using in vitro permeability assays and in vivo extravasation mice models show Rcan 1 as a stabilizer of the endothelial barrier or as a cellular dilator agent. Our studies demonstrate that histamine-induced Rcan 1 contributes to the stability of the endothelial barrier and also indicate that this mechanism is a late-secondary response to prevent the loss of fluids and/or the regulation of vascular tone.

Our studies demonstrate that exogenous overexpression of Rcan1 downregulates the expression of calcineurin-related genes such as COX2, NOS3, and CaMKII. H1R stimulation increases NOS3 synthesis through a mechanism that involves CaMKII in human vascular ECs $(24,50)$. NOS3 upregulation and consequent $\mathrm{NO}$ production is protective under normal conditions, though may be deleterious in a model of experimental anaphylaxis $(51,52)$. Our studies correlate the stability of the endothelial barrier with a decreased NOS 3 expression in Rcan 1 overexpressed cells, as well as a tendency to prevent the increase of NOS3 induced by histamine, suggesting a protective vasodilator role for Rcan1 in ECs. Interestingly, CaMKII has been reported as a suppressor of intracellular cAMP accumulation (53). The inhibited CaMKII may induce PKA-mediated responses, increasing the levels of cAMP, which in turn could contribute to barrier stability. In fact, direct evidence has demonstrated the ability of Rcan 1 to increase the phosphorylation of cAMP response element-binding protein through the negative regulation of the calcineurin signaling pathway (54). Accordingly, Rcan 1 overexpression decreases CaMKII levels in our EC system, supporting its role in the strengthening of the endothelial barrier. 


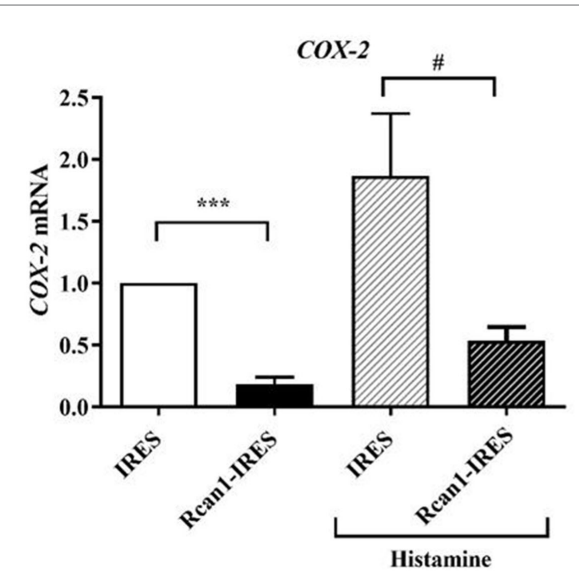

RCANI

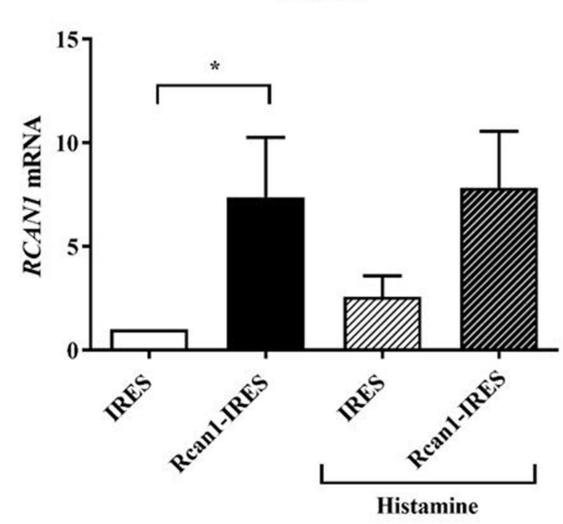

CaMKII

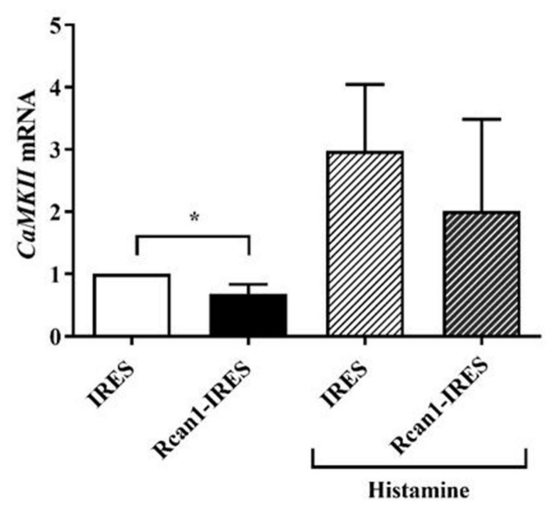

$M L C K$



NOS3

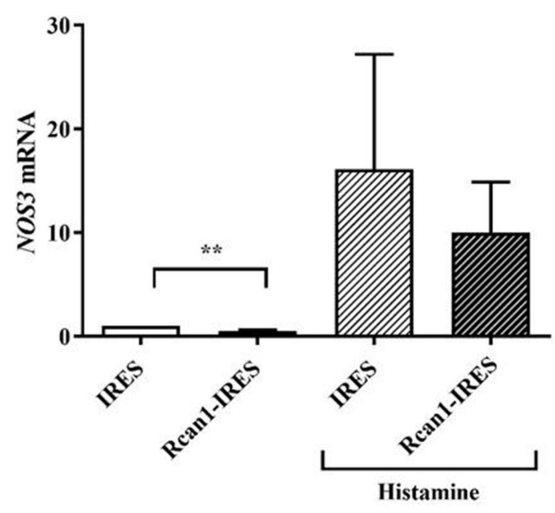

ROCK



FIGURE 5 | Regulator of calcineurin 1 (Rcan1)-IRES-GFP inhibits COX2, CaMKII, and nitric oxide synthase 3 (NOS3) expression in human vein (HV)-endothelial cells (ECS). Figure shows the qPCR analysis of COX2, CaMKII, NOS3, MLC-kinase (MLCK), ROCK1, and RCAN1 mRNA of IRES-GFP and Rcan1-IRES-GFP HV-ECs treated or not with Hist $1 \mu \mathrm{M}$ for $60 \mathrm{~min}$. Values represent amounts of mRNA normalized to the endogenous gene. Unpaired $t$-test analysis was applied to untreated or histamine-treated cells. Data represent means \pm SEM of duplicates determined by qPCR from indicated independent experiments: COX-2; ${ }^{\star \star \star \star} P<0.0001$, Rcan1-IRES-GFP control ( $n=13$ ) vs IRES-GFP control $(n=12)$; $P=0.0130$, Rcan1-IRES-GFP histamine $(n=14)$ vs IRES-GFP histamine $(n=15)$; CaMKII; ${ }^{\star} P=0.0454$, Rcan1-IRES-GFP control $(n=9)$ vs IRES-GFP control $(n=8)$; Rcan1-IRES-GFP histamine $(n=6)$ vs IRES-GFP histamine $(n=6) ;$ NOS3; ${ }^{\star *} P=0.0044$, Rcan1-IRES-GFP control $(n=6)$ vs IRES-GFP control $(n=6)$; Rcan1-IRES-GFP histamine $(n=5)$ vs IRES-GFP histamine $(n=5)$; RCAN1; ${ }^{\star} P=0.0392$, Rcan1IRES-GFP control $(n=10)$ vs IRES-GFP control $(n=10)$; Rcan1-IRES-GFP histamine $(n=11)$ vs IRES-GFP histamine $(n=9)$; MLCK; Rcan1-IRES-GFP control $(n=9)$ vs IRES-GFP control $(n=10)$; Rcan1-IRES-GFP histamine $(n=8)$ vs IRES-GFP histamine $(n=6)$; ROCK1; Rcan 1 -IRES-GFP control $(n=10)$ vs IRES-GFP control $(n=11)$; Rcan1-IRES-GFP histamine $(n=11)$ vs IRES-GFP histamine $(n=9)$.

Molecular pathways based on disruption of cell-cell contacts and which are regulated by phosphorylation and de-phosphorylation of the myosin light chain are also relevant to this field of research $(17,55,56)$. Although RhoA and ROCK have been recently recognized as key targets mediating histamine-induced vascular leakage and anaphylactic shock (14), our results do not reveal significant differences in $M L C K / R O C K 1$ when Rcan1 is overexpressed in HV-ECs. Nevertheless, the identification of COX2 as a downstream target of the CN/Rcan1 molecular pathway is a meaningful observation, and one which supports previous studies (57). COX-2 expression is induced by inflammatory stimuli and other mediators in the vascular wall, such as histamine, generating prostaglandin I2, and E2 production in HA-ECs (58). Proinflammatory phospholipid-derived mediators are critical modulators of vascular tone in physiological and pathological situations. COX-2 production induced by histamine has been reported in mast cells $(19,20)$. In addition to its products, prostaglandin D2, leukotrienes, thromboxane A2, and PAF are released rapidly in anaphylactic events $(59,60)$. In our studies, COX2 mRNA was substantially decreased upon histamine stimulation in Rcan1 overexpressed HV-ECs, suggesting a major relevance of the axis histamine/Rcan1-4/COX2 in anaphylaxis. In general, it can be speculated that both cell types (endothelial and mast cells) may contribute to prostanoid/ eicosanoid generation, which greatly render the vasoconstrictor/ vasodilator effects that occur in anaphylaxis. Our findings suggest that, through endothelial Rcan 1 expression, histamine could also contribute to the regulation of important molecular pathways related to anaphylaxis, although further investigations need to be performed to confirm this.

Knowledge of the consequences of human Rcan 1 overexpression is limited to evidence of genetic dysfunctions secondary to 


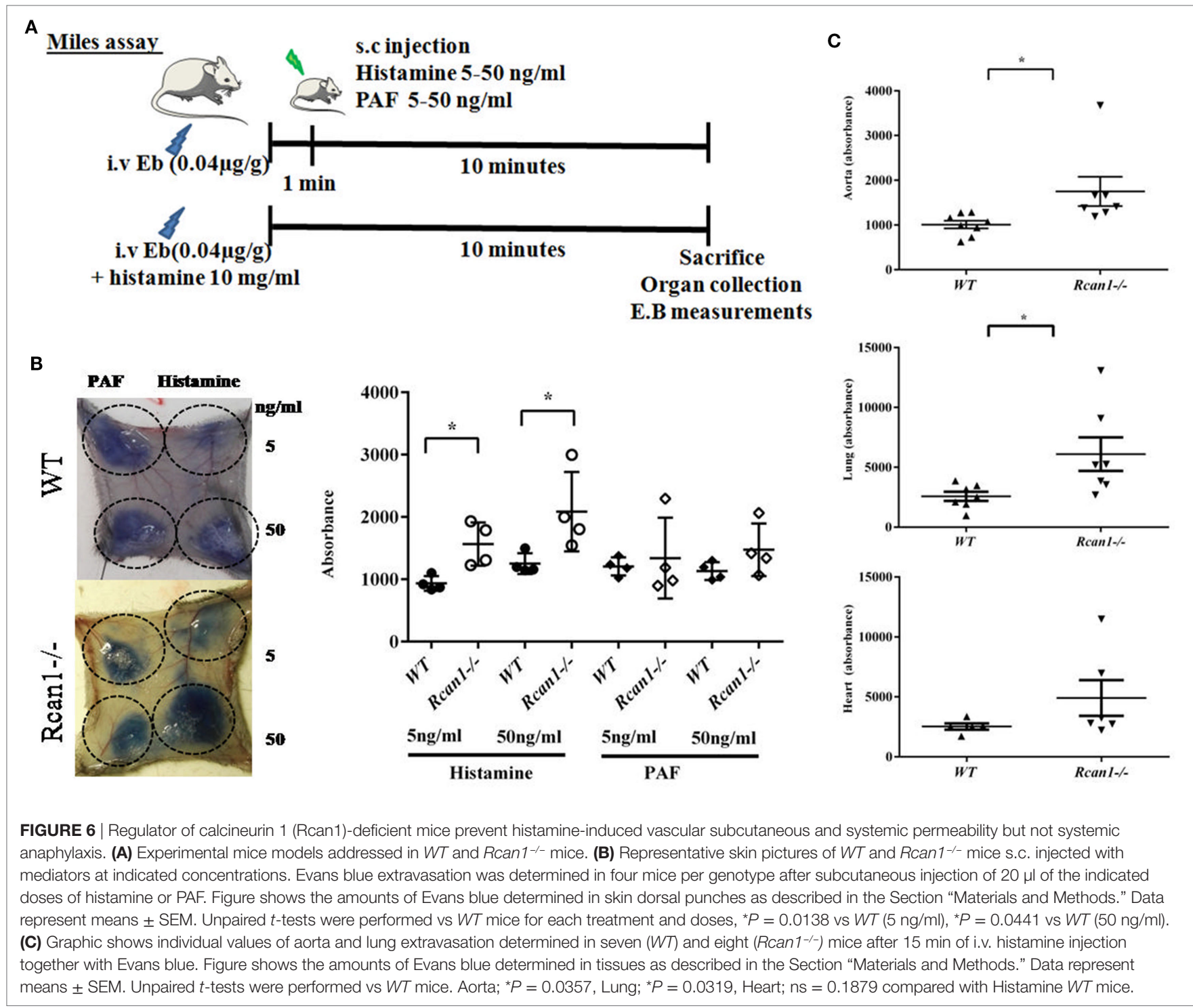

trisomy of chromosome 21 in patients with Down syndrome, who present defects of the immune system, though the prevalence of allergy is not high in these individuals (61). Previous studies using experimental anaphylaxis in Rcan1-deficient mice have found a role for Rcan 1 in regulating Fc- $\varepsilon$ RI-mediated signaling and mast-cell function (62). Rcan $1^{-/-}$bone-marrow-derived mast cells show increased transcriptional activation of NF- $\kappa \mathrm{B}$ and NFAT and calcineurin activity following stem-cell factor stimulation (63). On the other hand, Rcan 1 seems to be required for the development of pulmonary eosinophilia in allergic inflammation in mice (64). Additionally, Rcan1 plays a protective role for respiratory infections and sepsis in experimental mice models $(33,65)$. Consistent with this, studies performed on Rcan 1 transgenic mouse have found that Rcan 1 gives rise to cancer protection by inhibiting the calcineurin pathway in the vascular endothelium (66). Moreover, as an endogenous inhibitor of calcineurin, Rcan1 has been involved as a negative regulator in inflammatory molecular pathways belonging to ECs (47). Our results in human ECs and an experimental mice model support the immunosuppressive function of Rcan 1 for vascular permeability and anaphylaxis.

We, here, show that endothelial Rcan 1 maintains the integrity of the endothelial barrier in HV-ECs. This effect is closely correlated with the cellular dilatory process that must occur in the resident cells once the primordial effect of endothelial barrier breakdown has been induced by histamine. Extravasation or vascular permeability in micro-ECs may be best understood in terms of cellular contraction and dilatation, though cellular processes do not always correlate with physiology in vessels, and it is plausible that a similar process would occur in the context of anaphylaxis. Our results suggest that Rcan 1 overexpression produces cell dilation, at least in HV-ECs, which correlates with the stabilization of the HMVEC-D barrier.

In summary, this work contributes to the knowledge of anaphylaxis, showing endothelial Rcan1 as an inducible molecule which is modulated in the endothelial compartment 
upon contact with histamine through a calcineurin-sensitive pathway. Additionally, human functional assays and in vivo experimental mice models suggest a role for endothelial Rcan 1 controlling vascular permeability, most likely to recover the loss of fluids, and pointing to Rcan 1 as a plausible regulator of sensitivity to anaphylaxis in humans from the endothelial compartment.

\section{ETHICS STATEMENT}

The study was approved by the research ethics committees of the respective Gentofte and FJD hospitals, and written informed consent was obtained from all patients. Animal procedures were carried out in accordance with the European Union guidelines for the care and experimental use of animals. Protocols with reference PROEX: 391/15 received prior approval from the IIS-FJD Ethics Committee and the competent authorities in the region of Madrid.

\section{AUTHOR CONTRIBUTIONS}

CB-M, NM-B, and VE contributed to the performance of the experimental work; AM-Y and CP-V contributed to the performance of the experimental work included in the reviewed version. BMJ, LHG, LKP and VE as leaders of the overall project "molecular mechanisms in anaphylaxis”; LK, JC-H, and AG-C provided clinical support; NM-B and VE assisted in results interpretation;

\section{REFERENCES}

1. Sampson HA, Munoz-Furlong A, Campbell RL, Adkinson NF Jr, Bock SA, Branum A, et al. Second symposium on the definition and management of anaphylaxis: summary report - Second National Institute of Allergy and Infectious Disease/Food Allergy and Anaphylaxis Network symposium. J Allergy Clin Immunol (2006) 117(2):391-7. doi:10.1016/j.jaci.2005. 12.1303

2. Khan BQ, Kemp SF. Pathophysiology of anaphylaxis. Curr Opin Allergy Clin Immunol (2011) 11(4):319-25. doi:10.1097/ACI.0b013e3283481ab6

3. Joint Task Force on Practice Parameters; American Academy of Allergy, Asthma and Immunology; American College of Allergy, Asthma and Immunology; Joint Council of Allergy, Asthma and Immunology. The diagnosis and management of anaphylaxis: an updated practice parameter. J Allergy Clin Immunol (2005) 115(3 Suppl 2):S483-523. doi:10.1016/j.jaci. 2005.01.010

4. Brown SG. The pathophysiology of shock in anaphylaxis. Immunol Allergy Clin North Am (2007) 27(2):165-75,v. doi:10.1016/j.iac.2007.03.003

5. Finkelman FD, Khodoun MV, Strait R. Human IgE-independent systemic anaphylaxis. J Allergy Clin Immunol (2016) 137(6):1674-80. doi:10.1016/j. jaci.2016.02.015

6. Brown SG, Stone SF, Fatovich DM, Burrows SA, Holdgate A, Celenza A, et al. Anaphylaxis: clinical patterns, mediator release, and severity. J Allergy Clin Immunol (2013) 132(5):1141-9. doi:10.1016/j.jaci.2013.06.015

7. Vadas P, Perelman B, Liss G. Platelet-activating factor, histamine, and tryptase levels in human anaphylaxis. J Allergy Clin Immunol (2013) 131(1):144-9. doi:10.1016/j.jaci.2012.08.016

8. O'Mahony L, Akdis M, Akdis CA. Regulation of the immune response and inflammation by histamine and histamine receptors. J Allergy Clin Immunol (2011) 128(6):1153-62. doi:10.1016/j.jaci.2011.06.051

9. Panula P, Chazot PL, Cowart M, Gutzmer R, Leurs R, Liu WL, et al. International union of basic and clinical pharmacology. XCVIII. Histamine receptors. Pharmacol Rev (2015) 67(3):601-55. doi:10.1124/pr.114.010249
VE coordinated the work, designed the experiments, and wrote and edited the manuscript; NM-B, BMJ, MG-A, GA-L, CP-V, FV, JC-H, LH-G, and LKP reviewed the manuscript.

\section{ACKNOWLEDGMENTS}

This work was supported by the Spanish Council Ministry of Science and Innovation (Ramón y Cajal Program RyC-128802013), the Fundación Merck Salud, and the program LÓREAL for women in science 2016 (for VE). This work was also supported by grants from the Instituto de Salud Carlos III (PI13/00928 and PI16/00888) and RETIC ARADYAL (RD16/0006/0013, and RD16/0006/0003), co-supported by FEDER grants. NM-B is an investigator of Juan de la Cierva (FJCI-2014-20361). The authors want to thank Dr. J. M. Redondo for providing Rcan1-IRES-GFP lentiviral constructs and $R C A N 1^{-/-}$mice; Oliver Shaw for English editorial work; Prof. Per Stahl Skov and Dr. S. Falkencrone for supporting initial discussions; Andrea Quintana for technical support; Gonzalo Aldamiz-Echevarría, Angeles Heredero, and Ciro Baeza for their contributions from the cardiac and vascular surgery departments.

\section{SUPPLEMENTARY MATERIAL}

The Supplementary Material for this article can be found online at http://www.frontiersin.org/article/10.3389/fimmu.2017.01323/ full\#supplementary-material.
10. Lieberman P, Simons FE. Anaphylaxis and cardiovascular disease: therapeutic dilemmas. Clin Exp Allergy (2015) 45(8):1288-95. doi:10.1111/ cea. 12520

11. Brown SG, Blackman KE, Stenlake V, Heddle RJ. Insect sting anaphylaxis; prospective evaluation of treatment with intravenous adrenaline and volume resuscitation. Emerg Med J (2004) 21(2):149-54. doi:10.1136/ emj.2003.009449

12. Spindler V, Waschke J. Beta-adrenergic stimulation contributes to maintenance of endothelial barrier functions under baseline conditions. Microcirculation (2011) 18(2):118-27. doi:10.1111/j.1549-8719.2010.00072.x

13. Wawrzyniak M, Pich C, Gross B, Schutz F, Fleury S, Quemener S, et al. Endothelial, but not smooth muscle, peroxisome proliferator-activated receptor beta/delta regulates vascular permeability and anaphylaxis. J Allergy Clin Immunol (2015) 135(6):1625-35. doi:10.1016/j.jaci.2014.11.006

14. Mikelis CM, Simaan M, Ando K, Fukuhara S, Sakurai A, Amornphimoltham P, et al. RhoA and ROCK mediate histamine-induced vascular leakage and anaphylactic shock. Nat Commun (2015) 6:6725. doi:10.1038/ncomms7725

15. Egawa G, Nakamizo S, Natsuaki Y, Doi H, Miyachi Y, Kabashima K. Intravital analysis of vascular permeability in mice using two-photon microscopy. Sci Rep (2013) 3:1932. doi:10.1038/srep01932

16. Curry FR, Adamson RH. Vascular permeability modulation at the cell, microvessel, or whole organ level: towards closing gaps in our knowledge. Cardiovasc Res (2010) 87(2):218-29. doi:10.1093/cvr/cvq115

17. Dejana E, Orsenigo F. Endothelial adherens junctions at a glance. J Cell Sci (2013) 126(Pt 12):2545-9. doi:10.1242/jcs.124529

18. Simmons DL, Botting RM, Hla T. Cyclooxygenase isozymes: the biology of prostaglandin synthesis and inhibition. Pharmacol Rev (2004) 56(3):387-437. doi:10.1124/pr.56.3.3

19. Krystel-Whittemore M, Dileepan KN, Wood JG. Mast cell: a multi-functional master cell. Front Immunol (2015) 6:620. doi:10.3389/fimmu.2015.00620

20. Boyce JA. Mast cells and eicosanoid mediators: a system of reciprocal paracrine and autocrine regulation. Immunol Rev (2007) 217:168-85. doi:10.1111/j. 1600-065X.2007.00512.x 
21. Kumar P, Shen Q, Pivetti CD, Lee ES, Wu MH, Yuan SY. Molecular mechanisms of endothelial hyperpermeability: implications in inflammation. Expert Rev Mol Med (2009) 11:e19. doi:10.1017/S1462399409001112

22. Crabtree GR, Olson EN. NFAT signaling: choreographing the social lives of cells. Cell (2002) 109(Suppl):S67-79. doi:10.1016/S0092-8674(02)00699-2

23. Pan MG, Xiong Y, Chen F. NFAT gene family in inflammation and cancer. Curr Mol Med (2013) 13(4):543-54. doi:10.2174/1566524011313040007

24. Liu J, Farmer JD Jr, Lane WS, Friedman J, Weissman I, Schreiber SL. Calcineurin is a common target of cyclophilin-cyclosporin A and FKBP-FK506 complexes. Cell (1991) 66(4):807-15. doi:10.1016/0092-8674(91)90124-H

25. Davies KJ, Ermak G, Rothermel BA, Pritchard M, Heitman J, Ahnn J, et al. Renaming the DSCR1/Adapt78 gene family as RCAN: regulators of calcineurin. FASEB J (2007) 21(12):3023-8. doi:10.1096/f.06-7246com

26. Fuentes JJ, Pritchard MA, Estivill X. Genomic organization, alternative splicing, and expression patterns of the DSCR1 (down syndrome candidate region 1) gene. Genomics (1997) 44(3):358-61. doi:10.1006/geno.1997.4866

27. Genesca L, Aubareda A, Fuentes JJ, Estivill X, de la Luna S, Perez-Riba M. Phosphorylation of calcipressin 1 increases its ability to inhibit calcineurin and decreases calcipressin half-life. Biochem J (2003) 374(Pt 2):567-75. doi:10.1042/bj20030267

28. Saenz GJ, Hovanessian R, Gisis AD, Medh RD. Glucocorticoid-mediated co-regulation of RCAN1-1, E4BP4 and BIM in human leukemia cells susceptible to apoptosis. Biochem Biophys Res Commun (2015) 463(4):1291-6. doi:10.1016/j.bbrc.2015.06.106

29. Ermak G, Hench KJ, Chang KT, Sachdev S, Davies KJ. Regulator of calcineurin (RCAN1-1L) is deficient in Huntington disease and protective against mutant huntingtin toxicity in vitro. J Biol Chem (2009) 284(18):11845-53. doi:10.1074/jbc.M900639200

30. Fuentes JJ, Genesca L, Kingsbury TJ, Cunningham KW, Perez-Riba M, Estivill X, et al. DSCR1, overexpressed in down syndrome, is an inhibitor of calcineurin-mediated signaling pathways. Hum Mol Genet (2000) 9(11): 1681-90. doi:10.1093/hmg/9.11.1681

31. Minami T. Calcineurin-NFAT activation and DSCR-1 auto-inhibitory loop: how is homoeostasis regulated? J Biochem (2014) 155(4):217-26. doi:10.1093/ $\mathrm{jb} / \mathrm{mvu} 006$

32. Torac E, Gaman L, Atanasiu V. The regulator of calcineurin (RCAN1) an important factor involved in atherosclerosis and cardiovascular diseases development. J Med Life (2014) 7(4):481-7.

33. Minami T, Yano K, Miura M, Kobayashi M, Suehiro J, Reid PC, et al. The down syndrome critical region gene 1 short variant promoters direct vascular bed-specific gene expression during inflammation in mice. J Clin Invest (2009) 119(8):2257-70. doi:10.1172/JCI35738

34. Minami T, Horiuchi K, Miura M, Abid MR, Takabe W, Noguchi N, et al. Vascular endothelial growth factor- and thrombin-induced termination factor, Down syndrome critical region-1, attenuates endothelial cell proliferation and angiogenesis. J Biol Chem (2004) 279(48):50537-54. doi:10.1074/jbc. M406454200

35. Minami T, Miura M, Aird WC, Kodama T. Thrombin-induced autoinhibitory factor, Down syndrome critical region-1, attenuates NFAT-dependent vascular cell adhesion molecule-1 expression and inflammation in the endothelium. J Biol Chem (2006) 281(29):20503-20. doi:10.1074/jbc.M513112200

36. Esteban V, Mendez-Barbero N, Jimenez-Borreguero LJ, Roque M, Novensa L, Garcia-Redondo AB, et al. Regulator of calcineurin 1 mediates pathological vascular wall remodeling. J Exp Med (2011) 208(10):2125-39. doi:10.1084/ jem. 20110503

37. Mendez-Barbero N, Esteban V, Villahoz S, Escolano A, Urso K, Alfranca A, et al. A major role for RCAN1 in atherosclerosis progression. EMBO Mol Med (2013) 5(12):1901-17. doi:10.1002/emmm.201302842

38. Korhonen H, Fisslthaler B, Moers A, Wirth A, Habermehl D, Wieland T, et al. Anaphylactic shock depends on endothelial Gq/G11. J Exp Med (2009) 206(2):411-20. doi:10.1084/jem.20082150

39. Porta S, Serra SA, Huch M, Valverde MA, Llorens F, Estivill X, et al. RCAN1 (DSCR1) increases neuronal susceptibility to oxidative stress: a potential pathogenic process in neurodegeneration. Hum Mol Genet (2007) 16(9): 1039-50. doi:10.1093/hmg/ddm049

40. Khodoun MV, Strait R, Armstrong L, Yanase N, Finkelman FD. Identification of markers that distinguish IgE- from IgG-mediated anaphylaxis. Proc Natl Acad Sci U S A (2011) 108(30):12413-8. doi:10.1073/ pnas. 1105695108
41. Gill P, Jindal NL, Jagdis A, Vadas P. Platelets in the immune response: revisiting platelet-activating factor in anaphylaxis. J Allergy Clin Immunol (2015) 135(6):1424-32. doi:10.1016/j.jaci.2015.04.019

42. Simons FE, Ebisawa M, Sanchez-Borges M, Thong BY, Worm M, Tanno LK, et al. 2015 update of the evidence base: World Allergy Organization anaphylaxis guidelines. World Allergy Organ J (2015) 8(1):32. doi:10.1186/ s40413-015-0080-1

43. Aird WC. Phenotypic heterogeneity of the endothelium: I. Structure, function, and mechanisms. Circ Res (2007) 100(2):158-73. doi:10.1161/01. RES.0000255691.76142.4a

44. Dyer LA, Patterson C. Development of the endothelium: an emphasis on heterogeneity. Semin Thromb Hemost (2010) 36(3):227-35. doi:10.1055/ s-0030-1253446

45. Adderley SP, Zhang XE, Breslin JW. Involvement of the H1 histamine receptor, p38 MAP kinase, myosin light chains kinase, and Rho/ROCK in histamineinduced endothelial barrier dysfunction. Microcirculation (2015) 22(4): 237-48. doi:10.1111/micc.12189

46. Rafii S, Butler JM, Ding BS. Angiocrine functions of organ-specific endothelial cells. Nature (2016) 529(7586):316-25. doi:10.1038/nature17040

47. Hesser BA, Liang XH, Camenisch G, Yang S, Lewin DA, Scheller R, et al. Down syndrome critical region protein 1 (DSCR1), a novel VEGF target gene that regulates expression of inflammatory markers on activated endothelial cells. Blood (2004) 104(1):149-58. doi:10.1182/blood-2004-01-0273

48. Ermak G, Sojitra S, Yin F, Cadenas E, Cuervo AM, Davies KJ. Chronic expression of RCAN1-1L protein induces mitochondrial autophagy and metabolic shift from oxidative phosphorylation to glycolysis in neuronal cells. J Biol Chem (2012) 287(17):14088-98. doi:10.1074/jbc.M111.305342

49. Moerman A, Anssens S, Lapage K, Martens T, De HS. Near-infrared spectroscopy reveals transient impairment of microvascular reactivity during severe acute anaphylaxis. J Cardiothorac Vasc Anesth (2016) 30(1):149-53. doi:10.1053/j.jvca.2015.02.016

50. Kou R, Greif D, Michel T. Dephosphorylation of endothelial nitric-oxide synthase by vascular endothelial growth factor. Implications for the vascular responses to cyclosporin A. J Biol Chem (2002) 277(33):29669-73. doi:10.1074/jbc.M204519200

51. Cauwels A, Janssen B, Buys E, Sips P, Brouckaert P. Anaphylactic shock depends on PI3K and eNOS-derived NO. J Clin Invest (2006) 116(8):2244-51. doi:10.1172/JCI25426

52. Duran WN, Breslin JW, Sanchez FA. The NO cascade, eNOS location, and microvascular permeability. Cardiovasc Res (2010) 87(2):254-61. doi:10.1093/ $\mathrm{cvr} / \mathrm{cvq} 139$

53. Mika D, Richter W, Conti M. A CaMKII/PDE4D negative feedback regulates cAMP signaling. Proc Natl Acad Sci U S A (2015) 112(7):2023-8. doi:10.1073/ pnas. 1419992112

54. Kim SS, Seo SR. The regulator of calcineurin 1 (RCAN1/DSCR1) activates the cAMP response element-binding protein (CREB) pathway. J Biol Chem (2011) 286(43):37841-8. doi:10.1074/jbc.M111.232165

55. Orsenigo F, Giampietro C, Ferrari A, Corada M, Galaup A, Sigismund S, et al. Phosphorylation of VE-cadherin is modulated by haemodynamic forces and contributes to the regulation of vascular permeability in vivo. Nat Commun (2012) 3:1208. doi:10.1038/ncomms2199

56. Rigor RR, Shen Q, Pivetti CD, Wu MH, Yuan SY. Myosin light chain kinase signaling in endothelial barrier dysfunction. Med Res Rev (2013) 33(5):911-33. doi: $10.1002 /$ med.21270

57. Canellada A, Ramirez BG, Minami T, Redondo JM, Cano E. Calcium/ calcineurin signaling in primary cortical astrocyte cultures: Rcan1-4 and cyclooxygenase-2 as NFAT target genes. Glia (2008) 56(7):709-22. doi:10.1002/ glia. 20647

58. Tan X, Essengue S, Talreja J, Reese J, Stechschulte DJ, Dileepan KN. Histamine directly and synergistically with lipopolysaccharide stimulates cyclooxygenase- 2 expression and prostaglandin $\mathrm{I}(2)$ and $\mathrm{E}(2)$ production in human coronary artery endothelial cells. J Immunol (2007) 179(11):7899-906. doi:10.4049/jimmunol.179.11.7899

59. Li X, Lu Y, Jin Y, Son JK, Lee SH, Chang HW. Curcumin inhibits the activation of immunoglobulin e-mediated mast cells and passive systemic anaphylaxis in mice by reducing serum eicosanoid and histamine levels. Biomol Ther (Seoul) (2014) 22(1):27-34. doi:10.4062/biomolther.2013.092

60. Ashraf M, Murakami M, Kudo I. Cross-linking of the high-affinity IgE receptor induces the expression of cyclo-oxygenase 2 and attendant 
prostaglandin generation requiring interleukin 10 and interleukin 1 beta in mouse cultured mast cells. Biochem J (1996) 320(Pt 3):965-73. doi:10.1042/ bj3200965

61. Ram G, Chinen J. Infections and immunodeficiency in down syndrome. Clin Exp Immunol (2011) 164(1):9-16. doi:10.1111/j.1365-2249.2011.04335.x

62. Yang YJ, Chen W, Edgar A, Li B, Molkentin JD, Berman JN, et al. Rcan1 negatively regulates $\mathrm{Fc}$ epsilonRI-mediated signaling and mast cell function. J Exp Med (2009) 206(1):195-207. doi:10.1084/jem.20081140

63. Wu Z, Li Y, Macneil AJ, Junkins RD, Berman JN, Lin TJ. Calcineurin-Rcan1 interaction contributes to stem cell factor-mediated mast cell activation. J Immunol (2013) 191(12):5885-94. doi:10.4049/jimmunol.1301271

64. Yang YJ, Macneil AJ, Junkins R, Carrigan SO, Tang JT, Forward N, et al. Regulator of calcineurin 1 (Rcan1) is required for the development of pulmonary eosinophilia in allergic inflammation in mice. Am J Pathol (2011) 179(3):1199-210. doi:10.1016/j.ajpath.2011.05.022

65. Junkins RD, Macneil AJ, Wu Z, McCormick C, Lin TJ. Regulator of calcineurin 1 suppresses inflammation during respiratory tract infections. J Immunol (2013) 190(10):5178-86. doi:10.4049/jimmunol.1203196
66. Baek KH, Zaslavsky A, Lynch RC, Britt C, Okada Y, Siarey RJ, et al. Down syndrome suppression of tumor growth and the role of the calcineurin inhibitor DSCR1. Nature (2009) 459(7250):1126-30. doi:10.1038/nature08062

Conflict of Interest Statement: Declarations of transparency and scientific rigor. This Declaration acknowledges that this paper adheres to the principles for transparent reporting and scientific rigor in preclinical research recommended by funding agencies, publishers, and other organizations engaged with supporting research. The authors declare no competing financial interests.

Copyright (C) 2017 Ballesteros-Martinez, Mendez-Barbero, Montalvo-Yuste, Jensen, Gomez-Cardenosa, Klitfod, Garrido-Arandia, Alvarez-Llamas, Pastor-Vargas, Vivanco, Garvey, Cuesta-Herranz, Poulsen and Esteban. This is an open-access article distributed under the terms of the Creative Commons Attribution License (CC BY). The use, distribution or reproduction in other forums is permitted, provided the original author(s) or licensor are credited and that the original publication in this journal is cited, in accordance with accepted academic practice. No use, distribution or reproduction is permitted which does not comply with these terms. 